...there

might be an

increased risk

of multiple

vertebral

fractures

in patients

who had

discontinued

denosumab
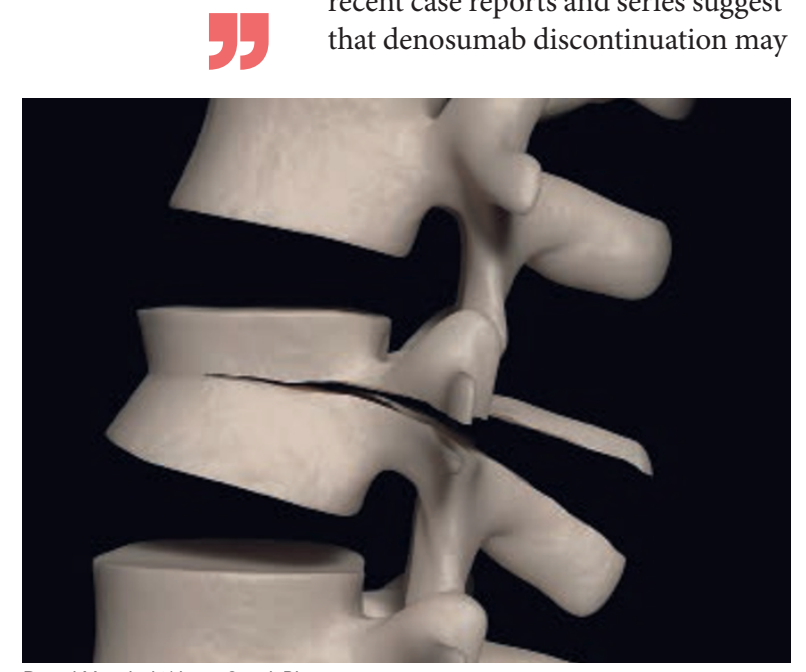

David Marchal/Alamy Stock Photo

The discontinuation of denosumab, a biologic therapy that targets receptor activator of NF- $\mathrm{B}$ ligand (RANKL; also known as TNFSF11), in the treatment of patients with osteoporosis has been discouraged in a new position paper from the European Calcified Tissue Society (ECTS).

"We noticed that there is a great need for advice on the duration of treatment with denosumab," states Carola Zillikens, corresponding author on the position paper. "Some recent case reports and series suggest that denosumab discontinuation may

OSTEOPOROSIS

\title{
Discontinuing denosumab discouraged
}

lead to an increased risk of multiple vertebral fractures," she explains. "In order to provide advice on management, ECTS formed a working group and reviewed existing literature on the effects of stopping denosumab."

Denosumab inhibits osteoclast function, thereby reducing bone resorption, but unlike some osteoporosis therapies, denosumab does not remain in the bone for long periods of time. The advice for most patients taking oral skeletally retained therapies, such as oral bisphosphonates, is to discontinue or have a break from taking the therapy after 5 years to avoid serious adverse effects. However, it seems that in clinical practice, these guidelines are also being applied to patients taking denosumab.

"Denosumab withdrawal quickly reverses the positive effects of the drug concerning bone mineral density, bone turnover markers and bone microarchitecture structure, and these changes may possibly be associated with an increased risk of multiple vertebral fractures," explains Elena Tsourdi, first author on the position paper. "This is a different situation from bisphosphonates, which have a persistent effect after discontinuation due to their high affinity for binding hydroxyapatite."

The ECTS working group evaluated data from 25 relevant publications, abstracts and clinical trials. In particular, data from the FREEDOM and FREEDOM Extension Trial indicated that there might be an increased risk of multiple vertebral fractures in patients who had discontinued denosumab compared with the placebo group.

"Based on available evidence, we advise that a re-evaluation should be performed after 5 years of denosumab treatment," says Zillikens. "Patients considered at high fracture risk should either continue denosumab therapy for up to 10 years or be switched to an alternative treatment. For patients at low risk, denosumab could be discontinued after 5 years but bisphosphonate therapy should be considered to reduce or prevent the rebound increase in bone turnover."

Joanna Collison

ORIGINAL ARTICLE Tsourdi, E. et al.

Discontinuation of denosumab therapy for osteoporosis: a systematic review and position statement by ECTS. Bone http://dx.doi. org/10.1016/j.bone.2017.08.003 (2017) 